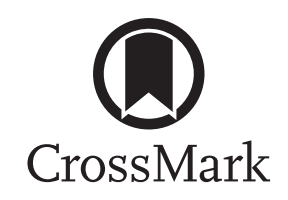

\title{
Excess economic burden of comorbidities in COPD: a 15-year population-based study
}

\author{
Wenjia Chen ${ }^{1}$, J. Mark FitzGerald ${ }^{2,3}$, Don D. $\operatorname{Sin}^{2,4}$ and Mohsen Sadatsafavi ${ }^{1,3}$ for \\ the Canadian Respiratory Research Network
}

\begin{abstract}
Affiliations: ${ }^{1}$ Collaboration for Outcomes Research and Evaluation, Faculty of Pharmaceutical Sciences, University of British Columbia, Vancouver, BC, Canada. ${ }^{2}$ Institute for Heart and Lung Health, Division of Respiratory Medicine, Faculty of Medicine, University of British Columbia, Vancouver, BC, Canada. ${ }^{3}$ Centre for Clinical Epidemiology and Evaluation, University of British Columbia, Vancouver, BC, Canada. ${ }^{4}$ Division of Respiratory Medicine, Dept of Medicine, The UBC Centre for Heart Lung Innovation, St Paul's Hospital, University of British Columbia, Vancouver, BC, Canada.
\end{abstract}

Correspondence: Don D. Sin, UBC Centre for Heart Lung Innovation, St Paul's Hospital, Providence Building, Room 8446, 1081 Burrard Street, Vancouver, BC V6Z 1Y6, Canada. E-mail: Don.Sinahli.ubc.ca

@ERSpublications

In patients with COPD, comorbidities cost twice that of COPD itself; cardiovascular diseases are most responsible http://ow.ly/95Ot30bfR9E

Cite this article as: Chen W, FitzGerald JM, Sin DD, et al. Excess economic burden of comorbidities in COPD: a 15-year population-based study. Eur Respir J 2017; 50: 1700393 [https://doi.org/10.1183/ 13993003.00393-2017].

ABSTRACT A better understanding of the true burden of chronic obstructive pulmonary disease (COPD) needs to consider the implications of comorbidities. This study comprehensively examined the impact of comorbidities on excess direct medical costs in COPD patients.

From health administrative data in British Columbia, Canada (1996-2012), we created a propensityscore-matched cohort of incident COPD patients and individuals without COPD. Health services use records were compiled into 16 major disease categories based on International Classification of Diseases codes. Excess costs (in 2015 Canadian dollars and converted to 2015 Euros; CAD1.000=EUR 0.706) were estimated as the adjusted difference in direct medical costs between the two groups.

The sample included 128424 subjects in each group. COPD patients generated excess costs of CAD5196/ EUR3668 per person-year (95\% CI CAD3540-8529), of which 26\% was attributable to COPD itself and 51\% was attributable to comorbidities (the remaining $23 \%$ could not be attributed to any specific condition). The major cost driver was excess hospitalisation costs. The largest components of comorbidity costs were circulatory diseases, other respiratory disorders, digestive disorders and psychological disorders (CAD696/ EUR491, CAD312/EUR220, CAD274/EUR193 and CAD249/EUR176 per person-year, respectively).

These findings suggest that the prevention and appropriate management of comorbidities in COPD patients may effectively reduce the overall burden of COPD.

This article has supplementary material available from erj.ersjournals.com

Received: Feb 232017 | Accepted after revision: April 182017

Support statement: This study was funded by the Canadian Respiratory Research Network (CRRN) and Genome Canada: Genome British Columbia. CRRN is supported by grants from the Canadian Institutes of Health Research (CIHR) - Institute of Circulatory and Respiratory Health, Canadian Lung Association/Canadian Thoracic Society, British Columbia Lung Association, and Industry Partners Boehringer Ingelheim Canada Ltd, AstraZeneca Canada Inc. and Novartis Canada Ltd. Funding for training of post-doctoral students and new investigators within the network was supported by the above funding sponsors and as well by GlaxoSmithKline Inc. The funders had no role in the study design, data collection and analysis or preparation of the manuscript. M. Sadatsafavi receives salary support from the Michael Smith Foundation for Health Research and CIHR. Funding information for this article has been deposited with the Crossref Funder Registry.

Conflict of interest: Disclosures can be found alongside this article at erj.ersjournals.com

Copyright @ERS 2017 


\section{Introduction}

Chronic obstructive pulmonary disease (COPD) is a leading cause of morbidity and mortality worldwide $[1,2]$. Patients with COPD are also more likely to suffer from comorbidities compared with individuals without COPD [3]. The increased prevalence can be caused by shared risk factors such as smoking [4]. Inflammation may also play a role as many comorbidities are associated with the activation of recognised inflammatory pathways $[5,6]$. Other comorbidities may be contributed by hypoxaemia or off-target effects of certain treatments (e.g. oral corticosteroids) $[5,6]$.

Comorbidities adversely impact the health of COPD patients, reducing their quality of life and increasing their risk for hospitalisations and death $[7,8]$. Therefore, comorbidities are likely to impose a significant economic burden on COPD patients. However, the magnitude of the burden is not well established. Previous studies on the economic burden of COPD comorbidities were focused on a limited number of comorbid conditions, mostly compared COPD patients with specific comorbid conditions with COPD patients without such conditions [9] and employed cross-sectional or longitudinal designs with very short follow-up, making it difficult to dynamically track the economic burden of comorbidities over time [9]. To the best of our knowledge, only two studies have explored the economic burden of COPD comorbidities from more than one disease domain $[10,11]$, and only one of these compared the costs between COPD patients and a non-COPD general population [10]. However, this study was based on only 1 year of follow-up.

Using 16 years of health administrative data from a large Canadian province, our current study estimated the incremental direct medical costs (referred to as "excess costs" for brevity) of comorbidities in COPD patients, over and beyond those incurred in a comparable non-COPD population. As a secondary objective, this study explored the impact of age, sex and comorbidity status at COPD onset on subsequent excess costs.

\section{Methods}

\section{Data source}

We used the provincial health administrative databases of British Columbia, Canada (with 4.4 million residents as of 2011 [12]) between January 1, 1996 and December 31, 2012 (University of British Columbia Human Ethics Certificate H13-00684). These databases provided linked, individual-level information on healthcare encounters of all legal British Columbia residents, including inpatient and outpatient services, dispensed prescription medications, community care services, demographics, vital statistics, and registration status (details of these databases are provided in supplementary appendix S1) [13-18]. All inferences, opinions and conclusions drawn in this study are those of the authors and do not reflect the opinions or policies of the Data Steward(s).

\section{Study design and sample}

This study has been registered in the European Network of Centres for Pharmacoepidemiology and Pharmacovigilance (EUPAS18242). We used a matched longitudinal study design by creating a cohort of patients with newly diagnosed COPD, and comparing their health services and medication utilisation to a comparable cohort of non-COPD individuals over the same period. A schematic presentation of the study design is provided in supplementary appendix S2.

The COPD cohort included individuals who satisfied a validated case definition of COPD [19], defined as the presence of one or more hospitalisations or two or more outpatient visits on different dates, with COPD as the most responsible diagnosis (International Classification of Diseases (ICD)-9: 491.xx, 492.xx, 493.2x, 496.xx; ICD-10: J43.xx, J44.xx) during any rolling 12-month period. This case definition, validated against chart reviews, has high sensitivity and specificity [19], and has been used in previous studies [20]. Once identified, we defined the index date as the next day after the patient first received a diagnosis of COPD (the choice of the "next" day was to avoid bias due to guaranteed resource use on the index date for the COPD cohort, which is not necessarily the case for the comparison group). The index date marked the beginning of the follow-up period. To ensure that we identified incident cases, we included only those patients who had been fully registered in the databases for at least 3 consecutive years prior to the index date. Patients also had to be registered for at least 1 year following the index date. To further improve the specificity of the COPD case definition, we restricted our analysis to patients who were aged $\geqslant 45$ years on their index date.

The non-COPD cohort was obtained as a random sample from the general population who had never been diagnosed with COPD and were matched to COPD patients with regard to sex, birth year, health service delivery area and neighbourhood income quintiles. Unlike in COPD patients for whom the index date was a specified date, the index date for the non-COPD cohort represented a random date during the study period. To maximise the possibility that COPD patients would find a match in the comparison 
group, we randomly assigned three index dates from the COPD cohort to each non-COPD person so that each potential control subject could have three chances to be matched to COPD patients. To create a cohort comparable to the COPD cohort, we excluded the index dates on which the individual in the comparison cohort was not fully registered in the databases for at least 3 years or was aged $<45$ years.

We then performed a multivariable logistic regression to calculate a propensity score, which was the probability that an individual belonged to the COPD cohort at baseline. Variables used for propensity score calculation included sociodemographic indicators as measured in the index year (age, sex, neighbourhood household income, health services delivery area), comorbidity status as measured in the 12 months prior to the index date (Charlson comorbidity index (CCI, excluding COPD), number of non-COPD hospitalisations, outpatient visits and medications), and the calendar year and season of the index date. Using a nearest-neighbourhood matching algorithm, we matched each person in the COPD cohort to a distinct person in the non-COPD cohort, with differences in their propensity score values of $\leqslant 0.01$.

\section{Outcome variables}

All costs were inflated to 2015 Canadian dollars using historical inflation rates that were obtained from the Consumer Price Index for British Columbia [21] and were converted to 2015 Euros $(\mathrm{CAD} 1.000=\mathrm{EUR} 0.706)$ [22]. The total medical costs were derived from four components: costs of inpatient encounters, outpatient encounters, filled prescriptions and use of community services (the latter included care at long-term care facilities, assisted living facilities, family care homes and group homes, adult day-care programmes, home care, and home supports). Using a case-mix methodology, inpatient costs were calculated by multiplying the resource intensity weight of each hospitalisation episode with the mean costs of hospitalisation in British Columbia during that fiscal year [23]. Costs of outpatient services, medications and community care were directly summed from the data. Emergency department visits were determined from the billing codes identifying emergency consultations and 1-day onsite hospital visits, and their costs were either captured by fee-for-service payments to physicians or by the corresponding inpatient records for those leading to an inpatient episode (22\% of all visits) [24].

All-cause medical costs were divided into costs attributable to COPD or comorbid conditions. COPD-attributable costs were calculated by attributing resource use records to a primary diagnosis of COPD. Comorbidity-attributable costs were calculated in the same fashion, with resource usage attributed to any of the 16 major comorbid areas in the ICD-10 system [25]. Some resource use records were associated with diagnoses of symptoms (ICD-10 code range R00-R69), which were also mapped to related comorbid categories. ICD-9 codes were used in the outpatient databases throughout the study period and in the inpatient databases up to 2002 (after which ICD-10 codes were used). We thus applied validated crosswalk tables to convert them into ICD-10 codes [26]. Medication records were also mapped to ICD-10 categories from the American Hospital Formulary Service (AHFS) major categories (supplementary appendix S3) [27]. For resource use records and medications that could not be associated with any disease or specified symptoms as described above (e.g. codes for injury, poisoning, burns and other external causes, and lab tests), as well as for miscellaneous ICD or AHFS codes, we grouped their related costs into a "nonattributable" category. Of note, community care costs were also grouped under the nonattributable category because the billing records were not associated with any ICD code.

\section{Statistical analysis}

All analyses were performed using SAS version 9.4 (SAS Institute, Cary, NC, USA). We compared the distribution of matching variables between COPD and non-COPD cohorts by visualising the distributions and by calculating the standardised difference for each variable (similarity defined as difference $\leqslant 0.10$ ) [28].

Excess costs were estimated as the adjusted difference in predicted costs of a COPD patient and their matched non-COPD subject. We estimated excess costs across conditions (COPD and 16 comorbid categories), as well as across specific cost components (hospitalisation, outpatient services, medications and community care). The unit of observation was person-year starting from the index date. To avoid underreporting bias when individuals were temporarily absent from the province (e.g. travelling outside of the province), we excluded person-years with $<300$ days of follow-up (unless death occurred in the year). Differences in excess costs across baseline age groups (45-54, 55-64 and $\geqslant 65$ years), sex and baseline comorbidity burden (none, CCI score 0 ; mild, CCI score 1 , moderate, CCI score 2; high, CCI score $\geqslant 3$ ) were examined using the regression coefficients of interaction terms between COPD and these three indicators. Since the first year of costs might be higher than subsequent years due to the acute treatment of COPD, we included a binary indicator for the first year. 
To accommodate zero-inflated and highly right-skewed cost data, we used a two-part generalised linear model. The first part was a logistic regression model to calculate the probability of incurring any costs. The second part was a gamma regression model with a logarithmic link to estimate the value of costs among patient-years with nonzero costs, which has been shown to perform well in the estimation of population-averaged healthcare costs [29]. The overall per-person-year costs were estimated as the probability of incurring any costs from the first part of model multiplied by the predicted costs for nonzero costs from the second part. Generalised Estimating Equations were applied to obtain valid inference for the clustered data around each matched pair. The adjusted effects of baseline risk factors on excess costs were estimated by combining the two parts using G-computation [30]. Inference (calculating p-values and confidence intervals) was performed with 50 rounds of bootstrapping. A detailed description of the estimation is provided in supplementary appendix S4.

\section{Results}

The study sample included 128424 incident COPD patients and an equal number of matched non-COPD individuals. Figure 1 shows the process of cohort selection. Baseline characteristics are shown in table 1. The mean age of COPD patients was 69.0 years and $49 \%$ were females. Matching was considered satisfactory as the standardised differences in the baseline matching variables were mostly between 0.00 and 0.05 (all $<0.10$ [28]). Residual imbalances were adjusted for by including the corresponding variables in subsequent multivariate models.

As shown in table 2, total adjusted excess direct medical costs were CAD5196/EUR3668 per person-year (95\% CI CAD3540-8529). Approximately 26\% (CAD1343/EUR948, 95\% CI CAD642-1878) and 51\% (CAD2673/EUR1887, 95\% CI CAD1822-4523) of the costs were related to COPD and to comorbidities, respectively. The remaining $23 \%$ of the excess costs could not be attributed to any specific condition $(3 \%$ community care costs and $20 \%$ nonattributable costs from outpatient services and medication dispensation records). Nonattributable costs were removed from subsequent analyses.

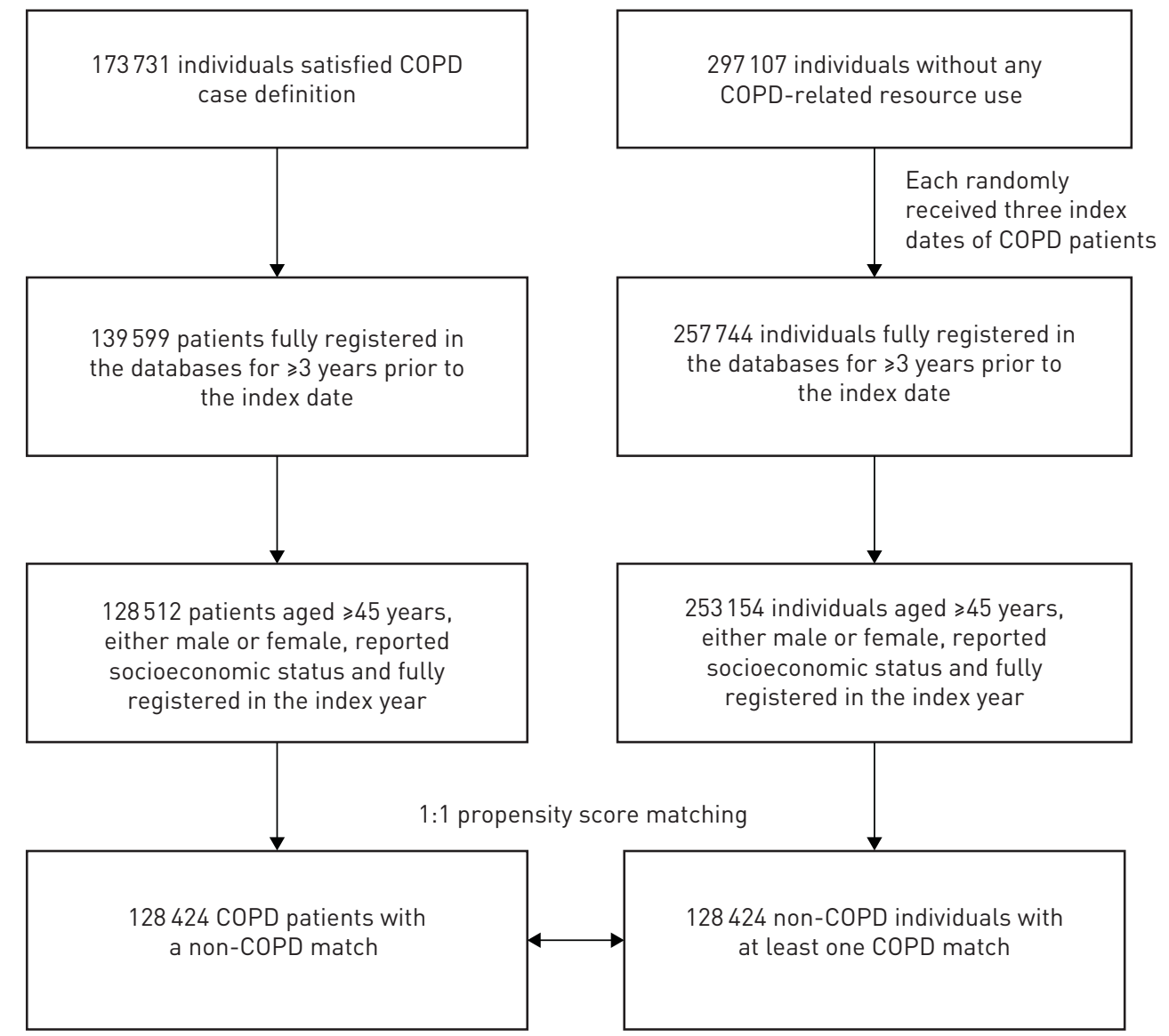

FIGURE 1 Cohort selection. COPD: chronic obstructive pulmonary disease. 
TABLE 1 Selected baseline characteristics of the propensity-score-matched study cohorts

\begin{tabular}{|c|c|c|c|}
\hline & $\begin{array}{l}\text { Non-COPD } \\
\text { individuals }\end{array}$ & $\begin{array}{l}\text { COPD } \\
\text { patients }\end{array}$ & $\begin{array}{l}\text { Standardised } \\
\text { difference }\end{array}$ \\
\hline Subjects & 128424 & 128424 & \\
\hline Age years" & $69.0 \pm 12.3$ & $69.4 \pm 11.8$ & 0.04 \\
\hline Sex & & & -0.02 \\
\hline Female & $61342(48)$ & $62359(49)$ & \\
\hline Male & $67082(52)$ & $66065(51)$ & \\
\hline $\begin{array}{l}\text { Neighbourhood household } \\
\text { income CAD }\end{array}$ & & & 0.01 \\
\hline First quintile & $34049(27)$ & $34068(27)$ & \\
\hline Second quintile & $27917(22)$ & $27677(22)$ & \\
\hline Third quintile & $24741(19)$ & $24530(19)$ & \\
\hline Fourth quintile & $22408(17)$ & $22564(18)$ & \\
\hline Fifth quintile & 19309 (15) & $19585(15)$ & \\
\hline Missing & Excluded $^{+}$ & Excluded $^{+}$ & \\
\hline Season of index date ${ }^{\pi}$ & & & 0.01 \\
\hline Spring & $27472(21)$ & $27627(22)$ & \\
\hline Summer & 34376 (27) & $34546(27)$ & \\
\hline Autumn & $35198(27)$ & $35018(27)$ & \\
\hline Winter & $31378(24)$ & $31233(24)$ & \\
\hline $\mathrm{CCI}$ (excluding COPD) $)^{\S}$ & $0.9 \pm 2.0$ & $1.0 \pm 1.5$ & 0.05 \\
\hline Non-COPD hospitalisation $\S$ & $0.2 \pm 0.8$ & $0.2 \pm 0.8$ & 0.01 \\
\hline Non-COPD physician visits ${ }^{\S}$ & $21.3 \pm 20.3$ & $22.0 \pm 19.5$ & 0.03 \\
\hline Non-COPD medications ${ }^{\S}$ & $39.0 \pm 107.5$ & $37.6 \pm 92.9$ & -0.01 \\
\hline
\end{tabular}

Data are presented as $\mathrm{n}$, mean \pm SD or $\mathrm{n}(\%)$, unless otherwise stated. COPD: chronic obstructive pulmonary disease; $\mathrm{CCl}$ : Charlson comorbidity index. ${ }^{\#}$ : difference in means or proportions divided by standard error (imbalance was defined as absolute value $>0.10$, comparing COPD cohort versus non-COPD cohort); $\rrbracket_{\text {: }}$ measured in the calendar year of index date; ${ }^{+}:<3 \%{ }^{\S}$ : measured in the 12 months prior to the index date.

As shown in figure 2, comorbidity-attributable excess costs were the highest for circulatory diseases (CAD696/EUR491, 95\% CI CAD296-1497), followed by respiratory disorders other than COPD (CAD312/EUR220, 95\% CI CAD172-854), digestive disorders (CAD274/EUR193, 95\% CI CAD106-516) and psychiatric disorders (CAD249/EUR176, 95\% CI CAD75-620). Infectious diseases (CAD194/EUR137, 95\% CI CAD108-385) and nervous system diseases (CAD144/EUR100, 95\% CI CAD11-300) were also associated with high excess costs. Overall excess costs for pregnancy/childbirth/puerperium, neoplasms or congenital abnormalities were not significantly different between COPD patients and non-COPD controls.

TABLE 2 Estimated excess costs in patients with chronic obstructive pulmonary disease (COPD) during the 10 -year follow-up period

\begin{tabular}{lcccc} 
& \multicolumn{3}{c}{ Excess costs (COPD versus non-COPD) } \\
\cline { 2 - 5 } & Overall & Hospitalisations & Outpatient visits & Medications \\
\hline \multirow{2}{*}{ All-cause medical costs } & $5196 / 3668$ & $2381 / 1681$ & $897 / 633$ & $1291 / 911$ \\
& $(3540-8529)$ & $(1130-4277)$ & $(677-1326)$ & $(957-1751)$ \\
COPD-attributable costs & $1343 / 948$ & $661 / 467$ & $98 / 69$ & $467 / 330$ \\
& $(642-1878)$ & $(193-1342)$ & $(43-249)$ & $(254-731)$ \\
Comorbidity-attributable costs \# & $2673 / 1887$ & $1290 / 911$ & $456 / 322$ & $689 / 486$ \\
& $(1822-4523)$ & $(662-2857)$ & $(344-717)$ & $(406-1054)$ \\
Nonattributable costs ${ }^{\Uparrow}$ & $1188 / 839$ & $450 / 318$ & $342 / 241$ & $135 / 95$ \\
& $(669-2374)$ & $(190-1008)$ & $(240-505)$ & $(73-235)$ \\
\hline
\end{tabular}

Data are presented as 2015 CAD/EUR per person-year (95\% Cl CAD); CAD1.000=EUR 0.706. " : the major comorbidity categories were defined by the International Classification of Diseases (ICD)-10 major disease categories and related symptoms [25]; " : includes community care costs, costs of general symptoms, signs and findings, injury, poisoning, burning and external causes, factors influencing health status, other special purposes, the American Hospital Formulary Service (AHFS) miscellaneous drug group, no ICD code, and no AHFS code. 


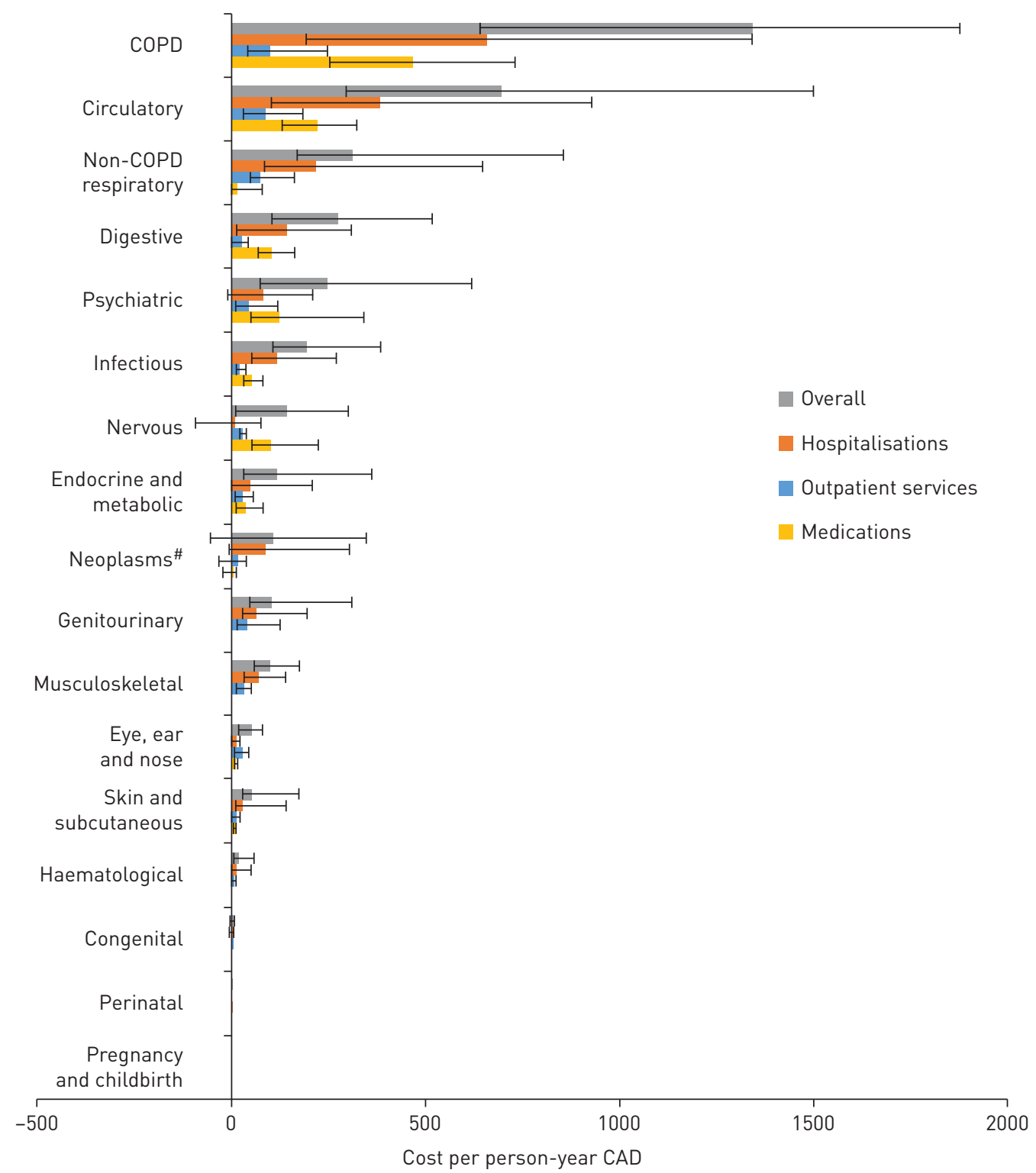

FIGURE 2 Estimated excess costs in patients with chronic obstructive pulmonary disease (COPD) during the 10-year follow-up period, by comorbid areas (CAD1.000=EUR0.706). "\#: costs of chemotherapy were not included in the PharmaNet medication costs [17].

Excess inpatient costs accounted for nearly half of COPD-attributable (49\%) and comorbidity-attributable (48\%) excess costs (table 2). Across all disease areas, excess inpatient costs remained the largest component except for psychiatric and nervous system diseases, and conditions of the eye, ear and nose, of which the latter were mainly driven by excess medication costs but barely incurred any excess hospitalisation costs (figure 2). Specifically, circulatory diseases (CAD383/EUR270), non-COPD respiratory disorders (CAD220/EUR155), digestive disorders (CAD142/EUR100) and infectious diseases (CAD118/EUR83) were associated with the highest excess inpatient costs. Excess medication costs were the highest for circulatory diseases (CAD224/EUR158), psychological disorders (CAD125/EUR88), digestive disorders (CAD104/EUR73) and nervous diseases (CAD103/EUR73). Additionally, excess outpatient costs were over CAD50/EUR35 per person-year for circulatory diseases (CAD88/EUR62) and non-COPD respiratory disorders (CAD76/EUR54).

Table 3 and supplementary figures S5-S7, respectively, show the estimated excess costs overall and for the most costly comorbidities across baseline risk groups. Comorbidity-attributable excess costs were about CAD250/EUR177 higher in women than in men, greatly increased from the subgroup with low baseline comorbidity burden (baseline CCI score $\leqslant 1$ ) to high burden (baseline CCI score $\geqslant 2$ ) and peaked in 
TABLE 3 Estimated excess costs due to chronic obstructive pulmonary disease (COPD) during the 10-year follow-up period, by risk factors at time of index hospitalisation

\begin{tabular}{|c|c|c|c|}
\hline \multirow[t]{2}{*}{ Baseline risk factors } & \multicolumn{3}{|c|}{ Excess costs (COPD versus non-COPD) } \\
\hline & All-cause & COPD-attributable & Comorbidity-attributable ${ }^{\#}$ \\
\hline \multicolumn{4}{|l|}{ Age years } \\
\hline \multirow[t]{2}{*}{$45-54$} & $3837 / 2709$ & $678 / 479$ & $2410 / 1701$ \\
\hline & $(2964-6228)$ & (399-1140) & $(1913-4436)$ \\
\hline \multirow[t]{2}{*}{$55-64$} & $4768 / 3366$ & $1125 / 794$ & $2660 / 1878$ \\
\hline & (3528-7497) & $(637-1770)$ & (2059-4898) \\
\hline \multirow[t]{2}{*}{$\geqslant 64$} & $4904 / 3462$ & $1367 / 965$ & $2369 / 1673$ \\
\hline & (3539-7730) & (905-2070) & (1462-4427) \\
\hline \multicolumn{4}{|l|}{ Sex } \\
\hline \multirow[t]{2}{*}{ Female } & $5016 / 3541$ & $1213 / 856$ & $2562 / 1809$ \\
\hline & (3190-7739) & (492-2020) & $(1773-4540)$ \\
\hline \multirow[t]{2}{*}{ Male } & $4456 / 3146$ & $1229 / 868$ & $2310 / 1631$ \\
\hline & (3027-7154) & (461-1998) & $(1442-4388)$ \\
\hline \multicolumn{4}{|l|}{ Comorbidity status ${ }^{\pi}$} \\
\hline \multirow[t]{2}{*}{ None } & $4213 / 2974$ & $1076 / 760$ & $2171 / 1533$ \\
\hline & (3054-6327) & $(461-1622)$ & (1831-3384) \\
\hline \multirow[t]{2}{*}{ Low } & $4366 / 3082$ & $1507 / 1064$ & $2028 / 1432$ \\
\hline & (3310-6953) & (706-2082) & (1438-3438) \\
\hline \multirow[t]{2}{*}{ Moderate } & $5908 / 4171$ & $1312 / 926$ & $3144 / 2220$ \\
\hline & (4253-10042) & (613-2020) & $(1943-5584)$ \\
\hline \multirow[t]{2}{*}{ High } & $6957 / 4912$ & $1365 / 964$ & $3915 / 2764$ \\
\hline & (3676-13701) & (639-2176) & (1137-8183) \\
\hline
\end{tabular}

Data are presented as 2015 CAD/EUR per person-year (95\% Cl CAD); CAD1.000=EUR 0.706. \#: the major comorbidity categories were defined by the International Classification of Diseases-10 major disease categories and related symptoms [25]; " : comorbidity status was measured by the Charlson comorbidity index (CCl, excluding COPD) in the 12 months prior to the index date (categorised as: none, $\mathrm{CCl}$ score 0 ; low, $\mathrm{CCl}$ score 1; moderate, $\mathrm{CCl}$ score 2; high, $\mathrm{CCl}$ score $\geqslant 3$ ).

patients aged 55-64 years. Age had a strong but opposite effect on excess costs of circulatory disease (higher among the elderly) and psychiatric disorders (higher among the younger individuals). The effect of baseline comorbidity was the strongest on excess costs of circulatory diseases, followed by infectious diseases. However, the effects of sex on the most costly comorbidities were relatively modest (supplementary figures S5-S7).

\section{Discussion}

Reliable estimates of the impact of comorbidities on COPD costs are needed to better understand the total burden of COPD. Such understanding is crucial for an unbiased evaluation of the cost-effectiveness of interventions (e.g. smoking cessation or improved physical activity) for COPD that have the potential to impact not only the lung but also other organs. We found that COPD patients incurred approximately CAD5196/EUR3668 excess costs per year, over and beyond the medical expenses of their counterparts in the general population who did not have COPD, although at baseline (i.e. before the diagnosis of COPD in the COPD cohort) the two groups had a similar pre-existing comorbidity profile. Given the total number of patients in the COPD cohort (128424), the excess economic burden of diagnosed COPD in British Columbia will be approximately CAD667/EUR471 million per year (for a population of 4.7 million people). Approximately half of such excess costs was related to comorbidities and a quarter to COPD itself (the rest was for community care or resource use records that could not be reliably attributed to any discrete condition). Inpatient costs were the main driver for COPD-attributable and most comorbidity-attributable excess costs. Circulatory diseases, respiratory diseases other than COPD, digestive disorders and psychiatric disorders were among the most costly comorbidities. In terms of risk factors, baseline comorbidity burden was found to strongly influence subsequent excess comorbidity costs, while age and sex also had a moderate effect which varied across individual comorbid conditions.

To the best of our knowledge, our study is the first to comprehensively examine the excess costs of comorbidities by comparing COPD patients to the general population over 16 years and attributing the incremental medical costs to one of the 16 major disease categories. This allowed us to directly compare across disease categories and explore the potential interconnection between COPD and respiratory and 
other extrapulmonary systems. Previous evidence on the excess costs of COPD comorbidities mostly compared medical costs between COPD patients with and without a discrete condition. Studies of this type comprise the majority of studies in a systematic review of comorbidity burden in COPD [9] and an updated study from ManNino et al. [11]. Our findings regarding the scale and distribution of comorbidity costs generally agree with these studies [9-11]. However, by focusing on a small number of discrete conditions, these studies did not fully capture the overall costs of comorbidities of COPD. Moreover, there was a lack of direct comparison with the general non-COPD population except for the study by LiN et al. [10], which had a relatively small sample size and a short follow-up period (1 year).

In our study, comorbidities related to the heart and lung, digestive system, and central nervous system had the greatest economic implication in patients with COPD. Apart from the effect of smoking on systemic diseases such as cardiovascular diseases, asthma and peptic ulcer bleeding [5], there is emerging evidence that systemic inflammation, oxidative stress and premature senescence may independently contribute to these diseases [31,32]. Findings of this study are particularly relevant to the industrialised world where population ageing offsets the benefits of decreased smoking on the future burden of COPD [33]. Aside from the shared risk factor and causal mechanisms, other, more specific mechanisms might explain the findings. For example, impairment in lung defence in COPD can lead to both acute and chronic infections [34], which in turn can result in chronic inflammation, acute bronchitis and community-acquired pneumonia. Use of inhaled corticosteroids to treat COPD can potentially blunt the immune system response to bacterial infection in the airways [34], which may increase the risk of pneumonia [35]. In addition, the substantial costs related to the central nervous system can point towards the often underappreciated systemic effect of COPD on the brain, such as hypoxaemia and its relationship to impaired cognitive function [36] as well as depression [37]. Potential mechanisms include neurological impairment due to systemic inflammation, hypoxia, oxidative stress [36], as well as inactivity, social isolation, and fear of dyspnoea and depression [37]. The reasons for psychiatric costs are, however, heterogeneous and further contributed to by stressors for patients dealing not only with COPD but also its multiple comorbidities [38].

Consistent with a recent study from Switzerland, we found inpatient costs to be the major component of COPD and most comorbidity-attributable excess costs except for psychiatric, nervous system diseases, and conditions of the eye, ear and nose [39]. Interactions between COPD and comorbidities might increase the risk and duration of hospitalisations [40]. Thus, reducing the burden of inpatient care by preventing acute exacerbations of COPD presents an important opportunity to lower the burden on patients.

Our results highlight the importance of comorbidities in determining the overall clinical benefits and cost-effectiveness of existing and novel therapeutics and management strategies in COPD. From a health economics perspective, preventive and therapeutic interventions and policies should be implemented only if they provide acceptable value for the resources they consume. A widely used metric to quantify this is the incremental cost-effectiveness ratio, which is compared to the decision maker's maximum acceptable cost to gain one unit of health benefit (e.g. in some jurisdictions, to be adopted, an intervention should cost less than CAD50000 for 1 quality-adjusted life year). As many interventions have the potential not only to impact COPD but also other organs (e.g. smoking cession, improving functional capacity or even inhaler therapies that reduce inflammation/risk of exacerbations thus indirectly affecting other systems), the evaluation of their cost-effectiveness requires valid estimates of the economic burden of comorbidity in COPD. The high economic burden of comorbidity in COPD means the estimates of the cost-effectiveness of such interventions can be significantly affected by considering how they impact the burden of COPD comorbidity.

This study has several limitations. First, the impact of COPD severity [41] on comorbidity costs was not assessed because information regarding pulmonary function measures was missing in the administrative health data. Second, misclassification bias can occur in the estimation of attributable costs when diagnostic codes were incorrectly assigned or the diagnosis itself was erroneous. Likewise, mapping prescription drugs to disease categories requires some degree of approximation. Additionally, we were unable to attribute certain utilisation records to a specific disease category due to missing or miscellaneous diagnostic information. Albeit not necessarily a limitation, our study did not adjust cost estimates using the survival probability for each follow-up year [42]. This is because we aimed to obtain population-averaged cost estimates that could be directly multiplied with COPD prevalence to derive the population burden of COPD at a cross-sectional time point. Lifetime and episode-of-care cost analysis was beyond the scope of our study.

In summary, in COPD patients, comorbidity-attributable costs were two times greater than those directly related to COPD. Among comorbidities, cardiovascular diseases imposed the greatest costs, followed by non-COPD respiratory diseases, digestive disorders and psychiatric disorders. Comorbidities impose a 
great economic burden for patients with COPD, and there is a pressing need to incorporate their impact on the cost-effectiveness evaluations of novel and existing therapeutics and management strategies in patients with COPD.

\section{Acknowledgements}

Author contributions: W. Chen had full access to all of the data in the study and takes responsibility for the content of the manuscript, including the integrity of the data and the accuracy of the data analysis. W. Chen, J.M. FitzGerald, D.D. Sin and M. Sadatsafavi conceived and designed the study. W. Chen analysed the data and wrote the first draft of the manuscript. W. Chen, J.M. FitzGerald, D.D. Sin and M. Sadatsafavi contributed to the interpretation of the data and clinical inputs. All authors were involved in the revision of the manuscript for important intellectual content and approved the final version to be published.

\section{References}

1 Buist AS, Vollmer WM, McBurnie MA. Worldwide burden of COPD in high- and low-income countries. Part I. The burden of obstructive lung disease (BOLD) initiative. Int J Tuberc Lung Dis 2008; 12: 703-708.

2 WHO. Burden of COPD. www.who.int/respiratory/copd/burden/en Date last accessed: November 8, 2016.

3 Mapel DW, Hurley JS, Frost FJ, et al. Health care utilization in chronic obstructive pulmonary disease. A case-control study in a health maintenance organization. Arch Intern Med 2000; 160: 2653-2658.

4 Vestbo J, Hurd SS, Agustí AG, et al. Global strategy for the diagnosis, management, and prevention of chronic obstructive pulmonary disease: GOLD executive summary. Am J Respir Crit Care Med 2013; 187: 347-365.

5 Hillas G, Perlikos F, Tsiligianni I, et al. Managing comorbidities in COPD. Int J Chron Obstruct Pulmon Dis 2015; 10: 95-109.

6 Gan WQ, Man SFP. Systemic effects and mortality in chronic obstructive pulmonary disease. BCMJ 2008; 50: $148-151$.

7 Sin DD, Anthonisen NR, Soriano JB, et al. Mortality in COPD: role of comorbidities. Eur Respir J 2006; 28: 1245-1257.

8 Huber MB, Wacker ME, Vogelmeier CF, et al. Comorbid influences on generic health-related quality of life in COPD: a systematic review. PLoS One 2015; 10: e0132670.

9 Huber MB, Wacker ME, Vogelmeier CF, et al. Excess costs of comorbidities in chronic obstructive pulmonary disease: a systematic review. PLoS One 2015; 10: e0123292.

10 Lin P-J, Shaya FT, Scharf SM. Economic implications of comorbid conditions among Medicaid beneficiaries with COPD. Respir Med 2010; 104: 697-704.

11 Mannino DM, Higuchi K, Yu T-C, et al. Economic burden of COPD in the presence of comorbidities. Chest 2015; 148: $138-150$.

12 Statistics Canada. Population by sex and age group, by province and territory (Number, both sexes). 2014. www. statcan.gc.ca/tables-tableaux/sum-som/101/cst01/demo31a-eng.htm Date last accessed: February 6, 2015.

13 British Columbia Ministry of Health. Consolidation File (MSP Registration \& Premium Billing). Population Data BC. Data Extract. MOH. 2014. www.popdata.bc.ca/data/internal/population/consolidationfile Date last accessed: February 15, 2017.

14 British Columbia Vital Statistics Agency. Vital Statistics Deaths. Population Data BC. Data Extract. 2014. www. popdata.bc.ca/data/internal/population/vsdeaths Date last accessed: February 15, 2017.

15 Canadian Institute for Health Information. Discharge Abstract Database (Hospital Separations). Population Data BC. Data Extract. MOH. 2014. www.popdata.bc.ca/data/internal/health/dad Date last accessed: February 15, 2017.

16 British Columbia Ministry of Health. Medical Services Plan (MSP) Payment Information File. Population Data BC. Data Extract. MOH. 2014. www.popdata.bc.ca/data/internal/health/msp Date last accessed: February 15, 2017.

17 British Columbia Ministry of Health. PharmaNet. Data Extract. Data Stewardship Committee. 2013. www.popdata. bc.ca/data/external/PharmaNet Date last accessed: February 15, 2017.

18 British Columbia Ministry of Health. Home and Community Care. Data Extract. MOH. 2014. www.popdata.bc.ca/ data/internal/health/hcc Date last accessed: February 15, 2017.

19 Gershon AS, Wang C, Guan J, et al. Identifying individuals with physician diagnosed COPD in health administrative databases. COPD 2009; 6: 388-394.

20 Khakban A, Sin DD, FitzGerald JM, et al. Ten-year trends in direct costs of COPD: a population-based study. Chest 2015; 148: 640-646.

21 Statistics Canada. Consumer Price Index, by province (monthly). 2015. www.statcan.gc.ca/tables-tableaux/ sum-som/101/cst01/cpis01a-eng.htm Date last accessed: July 30, 2015.

22 CanadianForex. Bank of Canada yearly average exchange rates back to 1990. www.canadianforex.ca/forex-tools/ historical-rate-tools/yearly-average-rates Date last accessed: April 6, 2017.

23 Poole B, Robinson S, MacKinnon M. Resource Intensity Weights and Canadian hospital costs: some preliminary data. Healthc Manag Forum 1998; 11: 22-26.

24 McKendry R, Reid RJ, McGrail KM, et al. Emergency Rooms in British Columbia: A Pilot Project to Validate Current Data and Describe Users. Vancouver, Centre for Health Services and Policy Research, 2002.

25 WHO. ICD-10: Version 2016. http://apps.who.int/classifications/icd10/browse/2016/en Date last accessed: October 24, 2015.

26 The National Bureau of Economic Research. CMS' ICD-9-CM to and from ICD-10-CM and ICD-10-PCS Crosswalk or General Equivalence Mappings. www.nber.org/data/icd9-icd-10-cm-and-pcs-crosswalk-generalequivalence-mapping.html Date last updated: May 11, 2016. Date last accessed: October 24, 2015.

27 McEvoy GK. AHFS Drug Information: Essentials 2006-2007. Bethesda, American Society of Health-System Pharmacists, 2006.

28 Cohen J. The $\mathrm{t}$ test for means. In: Statistical Power Analysis for the Behavioral Sciences. New York, Academic Press, 1977; pp. 19-74.

29 Malehi AS, Pourmotahari F, Angali KA. Statistical models for the analysis of skewed healthcare cost data: a simulation study. Health Econ Rev 2015; 5: 11. 
30 Austin PC, Urbach DR. Using G-computation to estimate the effect of regionalization of surgical services on the absolute reduction in the occurrence of adverse patient outcomes. Med Care 2013; 51: 797-805.

31 Huang KW, Luo JC, Leu HB, et al. Chronic obstructive pulmonary disease: an independent risk factor for peptic ulcer bleeding: a nationwide population-based study. Aliment Pharmacol Ther 2012; 35: 796-802.

32 Sin DD, Man SFP. Chronic obstructive pulmonary disease as a risk factor for cardiovascular morbidity and mortality. Proc Am Thorac Soc 2005; 2: 8-11.

33 Khakban A, Sin DD, FitzGerald JM, et al. The projected epidemic of COPD hospitalizations over the next 15 years: a population based perspective. Am J Respir Crit Care Med 2017; 195: 287-291.

34 Sethi S. Infection as a comorbidity of COPD. Eur Respir J 2010; 35: 1209-1215.

35 Calverley PMA, Anderson JA, Celli B, et al. Salmeterol and fluticasone propionate and survival in chronic obstructive pulmonary disease. N Engl J Med 2007; 356: 775-789.

36 Kent BD, Mitchell PD, McNicholas WT. Hypoxemia in patients with COPD: cause, effects, and disease progression. Int J Chron Obstruct Pulmon Dis 2011; 6: 199-208.

37 Lacasse Y, Rousseau L, Maltais F. Prevalence of depressive symptoms and depression in patients with severe oxygen-dependent chronic obstructive pulmonary disease. J Cardiopulm Rehabil 2001; 21: 80-86.

38 Norwood RJ. A review of etiologies of depression in COPD. Int J Chron Obstruct Pulmon Dis 2007; 2: 485-491.

39 Jansson S-A, Backman H, Rönmark E, et al. Hospitalization due to co-morbid conditions is the main cost driver among subjects with COPD - a report from the population-based OLIN COPD study. COPD 2015; 12: 381-389.

40 Chatila WM, Thomashow BM, Minai OA, et al. Comorbidities in chronic obstructive pulmonary disease. Proc Am Thorac Soc 2008; 5: 549-555.

41 Rabe KF, Hurd S, Anzueto A, et al. Global strategy for the diagnosis, management, and prevention of chronic obstructive pulmonary disease. Am J Respir Crit Care Med 2007; 176: 532-555.

42 Basu A, Manning WG. Estimating lifetime or episode-of-illness costs under censoring. Health Econ 2010; 19: $1010-1028$. 